\title{
Feeding dysfunctions and failure to thrive in neonates with congenital heart diseases
}

\author{
Giovanna Mangili, Elena Garzoli, Youcef Sadou \\ Local Health Unit Papa Giovanni XXIII, Bergamo, Italy
}

\begin{abstract}
Congenital heart disease (CHD) is the most common neonatal congenital malformation. The variety and severity of clinical presentation depend on the cardiac structures involved and their functional impact. The management of newborns with $\mathrm{CHD}$ requires a multidisciplinary approach, in which the nutritional aspect plays an important role. An adequate caloric intake during either preand post-surgical period, in fact, improves the outcome of these patients. In addition, the failure to thrive of these children in childhood has been related to long-term cognitive delay (attention deficit disorders, aggressive behaviour and poor social and emotional development). To date, there is a lack of standardized feeding protocols and caloric goals about how to feed neonates with $\mathrm{CHD}$, and current practice varies widely between centres. The latest American Society for Parenteral and Enteral Nutrition guidelines reiterate the importance of proteins, and recommend early start of enteral nutrition, also in the most severe heart diseases, such as univentricular forms. Necrotizing enterocolitis (NEC), the most frequent and feared complication of early feeding of these
\end{abstract}

Correspondence: Giovanna Mangili, Local Health Unit Papa Giovanni XXIII, Piazza OMS-Organizzazione Mondiale della Sanità 1, 24127, Bergamo, Italy.

Tel: +39.0352 .673163$

E-mail: gmangili@asst-pg23.it

Key words: Congenital heart disease; Newborn; Necrotizing enterocolitis; Minimal enteral feeding.

Contributions: the authors contributed equally.

Conflict of interest: the authors declare no potential conflict of interest.

Funding: funded by Merqurio Editore S.r.L, with the unconditional contribution of Nestlè Italiana S.p.A.

See online Appendix for additional materials.

Received for publication: 19 April 2018

Accepted for publication: 20 April 2018.

This work is licensed under a Creative Commons Attribution NonCommercial 4.0 License (CC BY-NC 4.0).

CC Copyright G. Mangili et al., 2018

Licensee PAGEPress, Italy

La Pediatria Medica e Chirurgica 2018; 40:196

doi:10.4081/pmc.2018.196 newborns, often represents an obstacle in spreading this practice. Furthermore, as demonstrated in premature infants, breastfeeding seems to reduce the incidence of NEC. That is why breastfeeding must be encouraged, even if it can be difficult for these mothers due to delivery complications, associated with infant disease. In addition, eating difficulties may persist even after discharge, because these patients require nutritional support through nasogastric tubes or percutaneous endoscopic gastrostomies.

\section{Introduction}

Congenital heart disease (CHD) is the most common congenital neonatal malformation with a reported prevalence between 4 and 10 per 1000 live births. ${ }^{1-4}$

The variety of cardiac defects is very wide because of the several pathologic combinations of different heart structures involved (atrias, ventricles, walls, large arteries, veins, valves).

To date, there is no unequivocal classification of congenital heart defects, since they can be categorized according to pathogenetic (pathogenetic classification), anatomic (anatomic classification) or functional criteria (clinical approach). This last one is the most used because the functional aspect of heart disease determines the therapeutic management at birth.

Clinical aspects of congenital heart defects vary from mild to critical. Severe CHD are lifethreatening and require immediate intervention. These forms, such as those requiring cardiac catheterization or surgery within the first year of life, represent $25 \%$ of all congenital heart disease. ${ }^{2}$ They are often ductus arteriosus (DA) dependent cardiopathies, because DA is the only way to ensure systemic and/or pulmonary perfusion.

Univentricular cardiopathies are the most complex among the severe forms. They are characterized by complete mixing, in atrias or ventricles, of systemic and pulmonary venous return (e.g. tricuspid atresia, hypoplastic left heart syndrome, heterotaxic conditions with asplenia or polysplenia). In these cases, the ventricular output is divided into two parallel circuits and due to complete blood mixing, oxygen saturations in aorta and in pulmonary artery are the same. Blood volume in each system depends on the resistances of the two circuits and on anatomical obstruction to pulmonary or systemic outflow.

Prognosis of newborns suffering from congenital heart disease, however, does not depend exclusively on the heart defect, but also on the presence of comorbidity (respiratory, gastrointestinal, renal, cerebral). After initial stabilization, these patients require a multidisciplinary approach, including proper nutritional management. Management of dysphagia, gastroesophageal reflux, intestinal malrotations combined with continuous enteral nutrition (both in pre- and post-operative period) and a tailored caloric 
intake can lead the newborn to surgery in good clinical conditions, reducing pre- and post-operative morbidity and mortality.

\section{Nutritional requirements in cardiopathic newborn}

Newborns affected by severe CHD may require an increased energy intake because, in addition to the physiological growth process, critical illnesses can lead to a risen protein catabolism and turnover. Failure to thrive of cardiopathic infants has been associated to long-term cognitive delay, including attention deficit disorders, aggressive behaviour and poor social and emotional development. ${ }^{5-7}$ Furthermore, malnutrition has shown to be associated with unfavorable clinical outcomes, both in pediatric and adult population. In fact, it has been associated with delayed wound healing, myocardial dysfunction, vascular endothelial damage, reduced muscular function and increased risk of post-operative infections (in particular pneumonia). 8,9

Mehrizi and Drash, already in 1962, showed that weight loss (prevalence of $55 \%$ ) was associated to an equally significant statural deficit (prevalence of 52\%) in cardiopathic newborns and highlighted the need of univocal recommendations for these patients. To date, there are still no guidelines on this topic. 10,11

It is well established that these patients, mainly those with univentricular cardiopathies, have high incidence of extrauterine growth retardation and a poor height-weight growth, despite having adequate weight for gestational age at birth. ${ }^{12}$ Weight deficit is the first and most severely involved, followed by a delay in statural and cranial circumference growth.

Although resting energy expenditure (REE) is normal or only moderately increased, overall energy expenditure - especially in critical illnesses - is significantly increased. Many factors are involved in this process: an increased metabolic demand due to heart failure (often associated with respiratory failure and therefore inadequate tissue oxygenation), surgical stress, malabsorption and suboptimal caloric intake. Furthermore, about $25 \%$ of cardiac newborns are small for gestational age, because of several reasons, such as genetic disorders. Associated genetic conditions such as Down syndrome, DiGeorge syndrome, Turner syndrome and trisomy 13 and 18, in fact, may influence energy intake, gastrointestinal absorption, expenditure and growth expectations. ${ }^{13}$

It is also important to consider that a low weight at the time of surgery affects an increased mortality in CHD children, and postoperative weight loss is a prognostic unfavorable index related to late mortality. ${ }^{14,15}$

There are several aspects and potential contraindications regarding nutrition of infants with CHD (Table 1).

Infants affected by CHD are often critical patients because they are also surgical patients. This leads to increased protein catabolism and turnover. The goals of nutrition in critically ill patients are to provide an adequate protein intake to facilitate

Table 1. Potential contraindications regarding nutrition of infants with congenital heart disease.

\begin{tabular}{ll} 
Gastrointestinal aspects & Low cardiac output \\
Gastrointestinal anatomic abnormality & Inotropic support \\
Maxillofacial abnormality & Tachycardia \\
\hline Increasing abdominal girth & Tachypnea \\
Excessive vomiting and/or diarrhoea & NIRS or MVO2 \\
\hline Guaiac stool & Lactic acidosis \\
Signs/symptoms of NEC &
\end{tabular}

wound healing, modulate the inflammatory response and preserve muscle mass. However, in infants with CHD the prevalence of malnutrition and chronic protein deficiency is documented in nearly half of the cases, respectively with only $68 \%$ and $40 \%$ of adequate energy and protein requirements at one week of life. 16

Currently, there are no clinical indicators or biochemical markers to define the REE and the optimal energy supplies to be provided in each case. The lastest American Society for Parenteral and Enteral Nutrition clinical guidelines for nutrition in the critically ill child set the protein requirement at $2-3 \mathrm{~g} / \mathrm{kg} / \mathrm{d}$ for infants 0 to 2 years of age. Preterm neonates have a greater protein requirement of $3.5-4 \mathrm{~g} / \mathrm{kg} / \mathrm{d}$ to promote growth during this critical period. ${ }^{17}$

More details on parental and enteral nutrition have been described in the Appendix.

\section{Necrotizing enterocolitis in cardiac newborn}

Concerns regarding the initiation of feeding in patients on prostaglandins, with low cardiac output, cyanosis or shunts are arisen by many clinicians for the risk of necrotizing enterocolitis (NEC) ${ }^{18-20}$ The incidence of NEC in neonates with CHD ranges from $1.62 \%$ to $7.8 \%$ and it may be due to an abnormal vascular flow in mesenteric vessels because of the underlying cardiac disease. ${ }^{20-22}$ In particular, patients with cyanogenic or single ventricle cardiomyopathy are at higher risk for NEC.

In preterm population, NEC typically develops at 2 to 4 weeks of age, while term infants with CHD are most likely to develop NEC in the first 7 days of life. However, disease localization to the terminal small bowel does not seem to differ between the two groups. ${ }^{23}$

It should be stressed that delay in oral feeding may increase the severity of NEC and can lead to intestinal mucosal atrophy with loss of barrier function. The postponed initiation of feeding may also worsen alteration of saprophytic flora (as much as during antibiotic therapy). On the other hand, early start of minimal enteral feeding (MEF), improves intestinal mucosa development and maturation of its associated immune system. ${ }^{24}$

Lyvonne and colleagues analysed the pre- and post-operative nutritional strategies in 59 cardiac children of pediatric and neonatal intensive care units from 18 European countries. They showed that in many units, pre-surgical enteral feeding is not routinely started. In one-third of the units, clinicians are uncomfortable in feeding patients while on prostaglandin infusion and there is a high concern about potential complications (first of all NEC), especially when nutrition is carried through an umbilical arterial catheter.

An optimal timing for beginning parenteral nutrition is not defined: it ranges from less than three days to a week. Moreover, regarding the post-operative period, there is no uniformity about contraindications to enteral feeding during therapy with vasoactive drugs, about the appropriate caloric target to provide and regarding indications to stop enteral nutrition (for example, for procedures such as cardiac catheterization or chest tubes or central lines placement). ${ }^{25}$ It is therefore evident how this lack of uniformity warrants further research in the next future.

\section{Breast milk nutrition}

Breast milk is considered the ideal nutrition for all newborns. The World Health Organization recommends exclusive breastfeeding up to 6 months of life because of proven nutritional, immunological and interpersonal (mother-child) benefits. Several studies 
showed the beneficial effects of breast milk in premature babies especially in reducing the incidence of NEC, while there is lack of evidence about the benefits of exclusive breastfeeding in newborns with CHD. 26

Furthermore, caloric intake of mother's milk may not be enough to support the growth of infants affected by CHD. Some studies, in fact, documented the use of formulas fortified with glucolipidic or glucidic components in this group of patients. ${ }^{27}$

An observational study of 122 newborns undergoing surgery for univentricular and biventricular cardiopathy, however, showed that postoperative growth and hospitalization are not influenced by the type of milk administered. 28

It is important to consider that the period following birth is extremely stressful for mothers and families. This can negatively influence mothers' ability to provide the baby her own milk. Of note, a recent study from the Children's Hospital in Philadelphia showed that breastfeeding of CHD infants can be successful if mothers receive prenatal lactation consultation focusing on the provision of human milk. ${ }^{29}$ Given the well-known nutritional and immunological role of human milk, donor milk is recommended as an alternative when mother production is not enough.

In conclusion, although there are no studies specifically focused on infants affected by congenital heart disease, it seems that human milk is the first choice also for these patients.

\section{Post-discharge nutrition and growth}

Nutritional issues of cardiopathic infants continue after hospital discharge. Difficulties in oral feeding, in fact, are frequent in these patients and can even get worse after cardiac surgery. Several studies documented a high rate of failure to thrive in infants with CHD after discharge, both in severe and milder forms. ${ }^{30,31}$

Although surgical repair of heart defects in the neonatal period usually results in weight gain within a few months, longitudinal growth and head circumference often experience delay for a year or more. Different causes contribute to this, such as persistent heart failure, aortic arch surgery, neurological damage, any associated genetic abnormalities, vocal cords injury by prolonged intubation, use of trans-oesophageal echocardiography, malabsorption, dysphagia, oral feeding aversion and gastroesophageal reflux. For these reasons, $44.6 \%$ of infants with CHD required feeding tubes at discharge because of a delay in full oral feeding attainment. ${ }^{32}$ Despite a more favourable feeding outcome in those with early beginning of oral feed, a significant percentage requires gavage feeding at discharge and about $20 \%$ of patients with hypoplastic left heart syndrome receive a percutaneous endoscopic gastrostomy. 33

The most effective method to ensure adequate nutritional intake is through continuous enteral feeding in 24 hours. At home it may be easier for parents to provide nutrition in 18-20 hours. The disadvantage of this type of tube feeding consists in poor oralmotor functions and in delayed progression of adequate oral intake. Several approaches can be used to combine oral feeding with tube feeding to provide adequate caloric intake and maintain

Table 2. Main post- discharge red flags.

1. Enteral intake $<100 \mathrm{ml} / \mathrm{kg} /$ die
2. Weight loss $>30 \mathrm{~g} /$ die
3. Failure to gain at least $20 \mathrm{~g}$ in 3 day

oral-motor feeding skills. One approach is to allow the infant to feed by nipple for a restricted period of time (generally 10-20 minutes) and then administer the remaining milk volume via the nasogastric tube. Another commonly used method is to encourage ad libitum oral feeding of breast milk (or fortified formula) during the day ( 8 to 12 hours), then provide the balance of the daily nutritional needs by continuous feeding during the night hours. ${ }^{34}$

It is important to perform close monitoring of growth and nutrition of infants affected by CHD both during hospitalization and after hospital discharge, especially in the first year of life, to promote adequate neurodevelopment. Some centres have established home monitoring programs with the aim of nutritional surveillance and management after discharge. ${ }^{35}$ Families are discharged home with weighing scale and trained to record daily weight. Critical situations suggestive for inadequate nutritional intake require a rapid clinical evaluation and are illustrated in Table 2 and indicated as red flags.

\section{Conclusions}

Infants affected by CHD have a greater risk of malnutrition and growth deficit, with negative long-term neurological outcomes. Currently, there are lack of evidence regarding nutritional approach in these patients and no guidelines available. However, literature highlights the importance of nutrition in the management of infants affected by CHD.

\section{References}

1. Ferencz C, Rubin JD, McCarter RJ, et al. Congenita heart disease: prevalence at livebirth. The Baltimore-Washington Infant Study. Am J Epidemiol 1985;121:31-6.

2. Hoffman JI, Kaplan S. The incidence of congenital heart disease. J Am Coll Cardiol 2002;39:1890-900.

3. Botto LD, Correa A, Erickson JD. Racial and temporal variations in the prevalence of heart defects. Pediatrics 2001;107:E32.

4. Talner CN. Report of the New England regional infant cardiac program, by Donald C. Fyler, MD, Pediatrics, 1980;65(suppl):375-461. Pediatrics 1998;102:258-9.

5. Black MM, Dubowitz H, Krishnakumar A, et al. Early intervention and recovery among children with failure to thrive: follow-up at age 8. Pediatrics 2007;120:59-69.

6. Dykman RA, Casey PH, Ackerman PT, et al. Behavioral and cognitive status in school-aged children with a history of failure to thrive during early childhood. Clin Pediatr (Phila) 2001;40:63-70.

7. Neubauer V, Griesmaier E, Pehbo"ck-Walser N, et al. Poor postnatal head growth in very preterm infants is associated with impaired neurodevelopment outcome. Acta Paediatr 2013;102:883-8.

8. Mikhailov TA, Kuhn EM, Manzi J, et al. Early enteral nutrition is associated with lower mortality in critically ill children. JPEN J Parenter Enteral Nutr 2014;38:459-66.

9. Agus MSD, Jaksic T. Nutritional support of the critically ill child. Curr Opin Pediatr 2002;14:470-81.

10. Floh AA, Slicker J, Schwartz SM. Nutrition and mesenteric issues in pediatric cardiac critical care. Pediatr Crit Care Med 2016;17:S243-9.

11. Mehrizi A, Drash A. Growth disturbance in congenital heart 
disease. J Pediatr 1962;61:418-29.

12. Mitting R, Marino L, Macrae D, et al. Nutritional status and clinical outcome in post-term neonates undergoing surgery for congenital heart disease. Pediatr Crit Care Med 2015;16:448-52.

13. Liechty EA. Growth and nutrition. In: Moller JH, O’Neal, eds. Cardiovascular disease in fetus and infant. New York, NY: Springer; 1989. pp 963-8.

14. Wong JJ, Cheifetz IM, Ong C, et al. Nutrition support for children undergoing congenital heart surgeries: A narrative review. World J Pediatr Congenit Heart Surg 2015;6:443-54.

15. Hehir DA, Easley RB, Byrnes J. Non cardiac challenges in the cardiac ICU: Feeding, growth and gastrointestinal complications, anticoagulation, and analgesia. World J Pediatr Congenit Heart Surg 2016;7:199-209.

16. Toole BJ, Toole LE, Kyle UG, et al. Perioperative nutritional support and malnutrition in infants and children with congenital heart disease. Congenit Heart Dis 2014;9:15-25.

17. Mehta NM, Compher C, ASPEN Board of Directors. A.S.P.E.N. Clinical Guidelines: nutrition support of the critically ill child. JPEN J Parenter Enteral Nutr 2009;33:260-76.

18. Larsen B, Goonewardene L, Field C, et al. Low energy intakes are associated with adverse outcomes in infants after open heart surgery. JPEN J Parenter Enteral Nutr 2013;37:254-60.

19. Curzon CL, Milford-Beland S, Li JS, et al. Cardiac surgery in infants with low birth weight is associated with increased mortality: analysis of the society of thoracic surgeons congenital heart database. J Thorac Cardiovasc Surg 2008;135:546-51.

20. McElhinney DB, Hedrick HL, Bush DM, et al. Necrotizing enterocolitis in neonates with congenital heart disease: Risk factors and outcomes. Pediatrics 2000;106:1080-7.

21. Mukherjee D, Zhang Y, Chang DC, et al. Outcomes analysis of necrotizing enterocolitis within 11958 neonates undergoing cardiac surgical procedures. Arch Surg 2010;145:389-92.

22. Dees E, Lin H, Cotton RB, et al. Outcome of preterm infants with congenital heart disease. J Pediatr 2000;137:653-9).

23. Cozzi C, Aldrink J, Nicol K, et al. Intestinal location of necro- tizing enterocolitis among infants with congenital heart disease. J Perinatol 2013;33:783-5.

24. Neu J, Walker WA. Necrotizing enterocolitis. N Engl J Med 2011;364:255-64.

25. Tume LN, Balmaks R, da Cruz E, et al. Enteral feeding practices in infants with congenital heart disease across European PICUs: a european society of pediatric and neonatal intensive care survey. Pediatr Crit Care Med 2018;19:137-44.

26. Sullivan S, Schanler RJ, Kim JH, et al. An exclusively human milk-based diet is associated with a lower rate of necrotizing enterocolitis than a diet of human milk and bovine milk-based products. J Pediatr 2010;156:562-7.e1.

27. Pillo-Blocka F, Adatia I, Shareef W, et al. Rapid advancement to more concentrated formula in infants after surgery for congenital heart disease reduces duration of hospital stay: a randomized clinical trial. J Pediatr 2004;145:761-6.

28. Rosti L, Vivaldo T, Butera G, et al. Postoperative nutrition of neonates undergoing heart surgery. Pediatr Med Chir 2011;33:236-40.

29. Torowicz DL, Seelhorst A, Froh EB, Spatz DL. Human milk and breastfeeding outcomes in infants with congenital heart disease. Breastfeed Med 2015;10:31-7.

30. Anderson JB, Marino BS, Irving SY, et al. Poor post-operative growth in infants with two-ventricle physiology. Cardiol Young 2011;21:421-9.

31. Medoff-Cooper B, Irving SY, Marino BS, et al. Weight change in infants with a functionally univentricular heart: from surgical intervention to hospital discharge. Cardiol Young 2011;21:136-44.

32. Kogon BE, Ramaswamy V, Todd K, et al. Feeding difficulty in newborns following congenital heart surgery. Congenit Heart Dis 2007;2:332-7.

33. Garcia X, Jaquiss RDB, Imamura M, et al. Preemptive gastrostomy tube placement after Norwood operation. J Pediatr 2011;159:602-7.e1.

34. Artman M, Mahony L, Teitel D. Neonatal cardiology. 3rd ed. New York, NY; 2017. pp 229-233.

35. Medoff-Cooper B, Ravishankar C. Nutrition and growth in congenital heart disease: a challenge in children. Curr Opin Cardiol 2013;28:122-9. 